Editorial

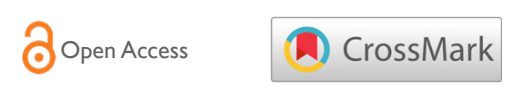

\title{
Night blindness may weaken your bones
}

\section{Editorial}

Nutritional night blindness is one of those old diseases humans have ever known. This disease has portrayals that revert to the ancient Egyptian medical papyri. At the end of First World War, a factor was identified in the liver that cures night blindness. Later on, this factor was named vitamin A. ${ }^{1}$ It is the precursor in the retina of the visual pigments of the rods and cones. ${ }^{2}$ The deficiency of vitamin A in diet causes retina to synthesize subnormal amount of visual pigments. This leads to visual sensitivity that constitutes night blindness. There are numerous experimental studies on night blindness of humans that depict a direct relationship with vitamin A. ${ }^{3}$ In subjects that have a deficiency of vitamin A, visual thresholds of both rods and cones began at once to rise establishes a mild night blindness. ${ }^{4,5}$ The threshold of vision returns to normal if vitamin A is administered within 2-3hours. However, some studies depicted two problems: Firstly, those subjects that have vitamin A deficient diet, their vision threshold increase once and remains unchanged from months to years. ${ }^{6-10}$ Secondly, subjects that develop night blindness may be cured within hours or took months to return to normal. Second problem raises other issues and complications. The visual pigments are composed of vitamin a aldehyde (retinene) joined to specific proteins of the rods and cones called "opsins." Limited amounts of visual pigment, formed in the normal retina, are due to opsins. In pasts, it was believed that the major cause of night blindness is vitamin A and less attention was paid to opsins. $^{2}$ If opsins are considered for their role in night blindness, then additional relationships may be established. The outer segment of rods and cones is composed of opsin, since the vitamin A chromophore constitutes only 1 per cent of this structure. However, this amount of opsin increases up to 40percent and 14 percent in frog and cattle rods ${ }^{2}$ respectively. Opsin is an essential constituent of rods and less likely to cones. Any change in this protein causes structural deterioration of visual receptors leading to blindness. Tansley $\mathrm{K}^{11,12}$ and Johnson $\mathrm{ML}^{13}$ confirmed these observations while working on rods and cones of rats and monkeys ${ }^{14}$ respectively. As the deficiency of vitamin A progresses, rods inside begin to degenerate and then the external limiting membrane, furthermore outer and inner nuclear layers. These changes flow from central to peripheral sections of the retina. With supplementation of vitamin A, degenerated rods are completely replaced within 10-18weeks of vitamin A supplementation. These observations conclude that time required to cure blindness depends upon the extent of retinal structure deteriorated by vitamin A. The deficiency of vitamin A may result in night blindness. However, its excess may be partly responsible for the rise of osteoporosis among older peoples. It may make your bones brittle. A study ${ }^{15}$ showed that excessive vitamin A supplementation increases the likelihood of bone fracture by seven times. Therefore, there is a need to monitor the current fortification levels. Previous studies have recommended that vitamin A plays a steady role in the weakening of bones. This weakening is mostly linked with age. However, the link between vitamin A and bone or hip fracture is established. Use of vitamin A is very common in Scandinavian countries and in the United States. In these countries, researchers found that excessive use of vitamin A has increased rates of bone fractures. Similarly, a study conducted by Swedish researchers found that men who had the highest levels of

\author{
Volume 5 Issue 4 - 2017
}

Tanveer Ahmed Khan

Rashid Latif College of Pharmacy, Pakistan

Correspondence: Tanveer Ahmed Khan, Rashid Latif College of Pharmacy, Pakistan, Tel +923202882884, Email tanveerahmedkhan754@gmail.com

Received: June 28, 2017 | Published: September 22, 2017

vitamin A at the start of the study were significantly more likely to suffer a bone or hip fracture, and the overall risk of fracture increased $64 \%$ in men with the highest levels compared with those with average levels. The hazard for fracture around the individuals with the most elevated levels of vitamin A might have been seven times higher over the individuals of those most reduced levels. ${ }^{15}$ In conclusion, we can say that there is a need to monitor intake of vitamin A for its deficiency in blindness. Otherwise, it's over intake may lead to weakening of bones.

\section{Acknowledgements}

None.

\section{Conflict of interest}

Author declares that there is no conflict of interest.

\section{References}

1. Mc Collum EV, Simmonds N. The dietary deficiencies of the white bean, Phaseolus vulgaris. J Biol Chem. 1917;29:521-536.

2. Wald G, John E Dowling. Vitamin A deficiency and night blindness. Proc Natl Acad Sci USA. 1958;44(7):648-661.

3. Wald G, Jeghers H, Arminio J. An Experiment in Human Dietary NightBlindness. Am J Physiol. 1938;123(3):732-746.

4. Schiotz H. Tonometrie. Arch Augenheilk. 1909;62:317-339.

5. Haig C, Hecht S, Patek AJ. Vitamin A and rod-cone dark adaptation in cirrhosis of the liver. Science. 1938;87(2267):534-536.

6. Steffens LF, Bair HL, Sheard C. Photometric measurements on visual adaptation in normal adults on diets deficient in vitamin A. Proceedings of Staff Meetings of the Mayo Clinic. 1939;14:698-704.

7. Hecht S, Mandelbaum J. Dark Adaptation and Experimental Human Vitamin A Deficiency. Am J Physiol. 1940;130(4):651-654.

8. Brenner S, Roberts LJ. Effects of Vitamin A depletion in Young Adults. Arch Intern Med. 1943;71(4):474-482.

9. Wald G, Brouhi L, Johnson RE. Experimental Human Vitamin A Deficiency and the Ability to Perform Muscular Exercise. Am J Physiol. 1942;137(3):551-556. 
10. Hume EM, Krebs HA. Vitamin A Requirements of Human Adults: An experimental study of vitamin A deprivation in man : a report of the Vitamin A Sub-Committee of the Accessory Food Factors Committee. JAMA. 1949;140(16):1310.

11. Tansley K. Factors Affecting the Development and Regeneration of Visual Purple in the Mammalian Retina. Proc Roy Soc London B. 1933;114(786):79-103.

12. Tansley K. The effect of vitamin A deficiency on the development of the retina and on the first appearance of visual purple. Biochem J. 1936;30(5):839-844.
13. Johnson ML. Degeneration and repair of the rat retina in Avitaminosis A. Arch Ophthalmol. 1943;29(5):793-810.

14. Ramingalaswami V, Leach EH, Sriramachari S. Ocular structure in vitamin A deficiency. Quart J Exptl Physiol. 1955;40(4):337-347.

15. Michaelsson MD, Lithell H, Vessby B, et al. Serum retinol levels and risk of fracture. N Engl J Med. 2003;348(4):287-294. 